\title{
Impact of Three-Dimensional Surgical Simulation on Pancreatic Surgery
}

\author{
Ryoichi Miyamoto Yukio Oshiro Ken Nakayama Nobuhiro Ohkohchi \\ Division of Gastroenterological and Hepatobiliary Surgery, and Organ Transplantation, \\ Department of Surgery, University of Tsukuba, Tsukuba, Japan
}

\section{Keywords}

Pancreatic surgery · Three-dimensional imaging · Surgical operation training

\begin{abstract}
Background/Aims: Anatomical variations are frequently encountered during hepato-biliarypancreatic surgeries, requiring surgeons to have a precise understanding of the surgical anatomy in order to perform a safe surgery. We evaluated the impact of novel three-dimensional (3D) surgical simulation on pancreatic surgeries to enhance surgical residents' understanding. Methodology: Between January 2013 and May 2014, 61 preoperative 3D surgical simulations were performed. The consistency (0-10, with 10 representing $100 \%$ consistency) among the 15 surgical residents' anatomical drawings from multidetector computed tomography images and the simulated 3D images by SYNAPSE VINCENT ${ }^{\circledR}$ was assessed. We divided the surgical residents into two groups - first- to fifth-year postgraduate doctors (group A) and sixthto tenth-year postgraduate doctors (group B) - and compared the self-assessment scores between these two groups. Results: In terms of the self-assessment scores, a statistically significant difference was observed between the two groups $(p<0.001)$. Conclusions: In this study, 3D surgical simulation was useful for preoperative assessments prior to pancreatic surgery, especially in younger postgraduate surgeons.

(C) 2017 S. Karger AG, Basel
\end{abstract}

\section{Introduction}

Anatomical variations are frequently encountered in hepato-biliary-pancreatic surgeries, requiring a precise understanding of the positional relationships among the lesions, surrounding organs, and vessels to perform a safe surgery $[1,2]$. In particular, surgical residents, who are less experienced surgeons, require repeated training to understand the three- 
dimensional (3D) anatomical relationships among these entities. In the past, surgeons assessed the consistency between the multidetector computed tomography (MDCT) images obtained before surgery and the actual anatomy observed during surgery. However, surgical residents often experience difficulties in accurately constructing the anatomy from twodimensional MDCT images. The development of recent imaging technology, such as 3D reconstruction software, aids in easy recognition of accurate 3D images. We focused on the use of 3D surgical simulations to share accurate information with the entire surgical staff, including assistants, and to recognize precise surgical anatomy [3-7].

Since 2010, we have constructed 3D images from MDCT datasets of patients prior to hepatic resection and shared the images with the surgical staff $[8,9]$. Furthermore, we were the first to develop novel 3D models by integrating MDCT and magnetic resonance cholangiopancreatography (MRCP) images, and we have extended the use of this simulation to biliary-pancreatic surgeries $[3,4,10]$. We evaluated the impact of novel 3D surgical simulation on pancreatic surgeries including pancreaticoduodenectomy (PD) and distal pancreatectomy (DP) to enhance surgical residents' understanding.

\section{Methodology}

Evaluation of 3D Images for Pancreatic Surgery

Sixty-one patients underwent pancreatectomy between January 2013 and May 2014. Preoperative 3D surgical simulations and navigation were performed. First, the 15 surgical residents ( 4 in their third postgraduate year, 4 in their fourth, 3 in their sixth, and 4 in their seventh) drew 3D image sketches from MDCT images. Furthermore, to evaluate the differences between the anatomical drawings that the surgical residents sketched and the simulated 3D images based on the number of years of postgraduate experience, selfdrawn sketches based exclusively on the MDCT images were compared with 3D simulated images in the preoperative conferences, which is supported by our Department. Finally, self-assessment scores, indicating the differences between the 3D images wherein a 10 on a 10-point scale indicated $100 \%$ consistency, were determined using questionnaires (Appendix). A total of 181 self-assessment questionnaire responses from all surgical residents were obtained. To assess whether the difference of the self-assessment scores was influenced by the number of postgraduate years, we divided the surgical residents into two groups - first- to fifthyear postgraduate doctors (group A) and sixth- to tenth-year postgraduate doctors (group B) - and compared the self-assessment scores between these two groups. When drawing their sketches, the surgical residents were instructed to pay particular attention to the following points: (a) the route of the arterial system, especially the hepatic artery, the splenic artery, and the divergent patterns of the inferior pancreaticoduodenal artery (IPDA); (b) the route of the venous system, especially the left gastric vein, the inferior mesenteric vein, and the divergent patterns of the gastro-colic trunk; and (c) the tumor location and dissection line of the pancreas. Two or more senior surgeons assessed the reproducibility of the sketches and questionnaire scores. The participants of the conference include surgical staff (including young surgeons), senior surgeons (including those who are specializing in pancreatic surgery), and medical students. The self-assessment by the young surgeons for the 3D anatomical structure was performed as a part of the preoperative conference. First, the consistency between the surgical residents' 3D drawing and the simulated 3D image was discussed by the participants. Thereafter, the young surgeons were asked to complete the questionnaire about the consistency between their 3D drawings and the simulated 3D images. This study was approved by the Ethics Committee of Tsukuba University Hospital.

\section{D Images Used in the Present Study}

We used the SYNAPSE VINCENT ${ }^{\circledR}$ medical imaging system (Fujifilm Medical Co., Ltd., Tokyo, Japan) to construct 3D images from MDCT images. We also developed 3D images by integrating MDCT and MRCP images to produce accurate preoperative anatomical images (Fig. 1). By integrating these two images, we were able to understand the anatomical relationships among the bile duct and the pancreatic duct, the vascular components, i.e. the hepatic artery and portal vein, and the parenchymal organs of the pancreas and liver. A preoperative conference based the 3D images also enabled the preoperative sharing of 3D anatomical images with the surgical staff $[3,4,10]$. 


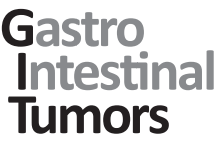

Fig. 1. Three-dimensional (3D) images of pancreatic tumors obtained by integrating multidetector computed tomography and magnetic resonance cholangiopancreatography images. This image represents the 3D image view from the front side of the patient. The red color represents the arteries; blue represents the veins, including the portal vein; light blue represents the inferior vena cava; green represents the biliary duct; turquoise represents the pancreatic duct; and pink represents the pancreatic tumor.

\begin{tabular}{l|l}
\hline Gastrointest Tumors 2017;4:84-89 \\
\hline DOI: 10.1159/000484894 & $\begin{array}{l}\text { @ 2017 S. Karger AG, Basel } \\
\text { www.karger.com/gat }\end{array}$ \\
\hline
\end{tabular}

Miyamoto et al.: Impact of Three-Dimensional Surgical Simulation on Pancreatic Surgery

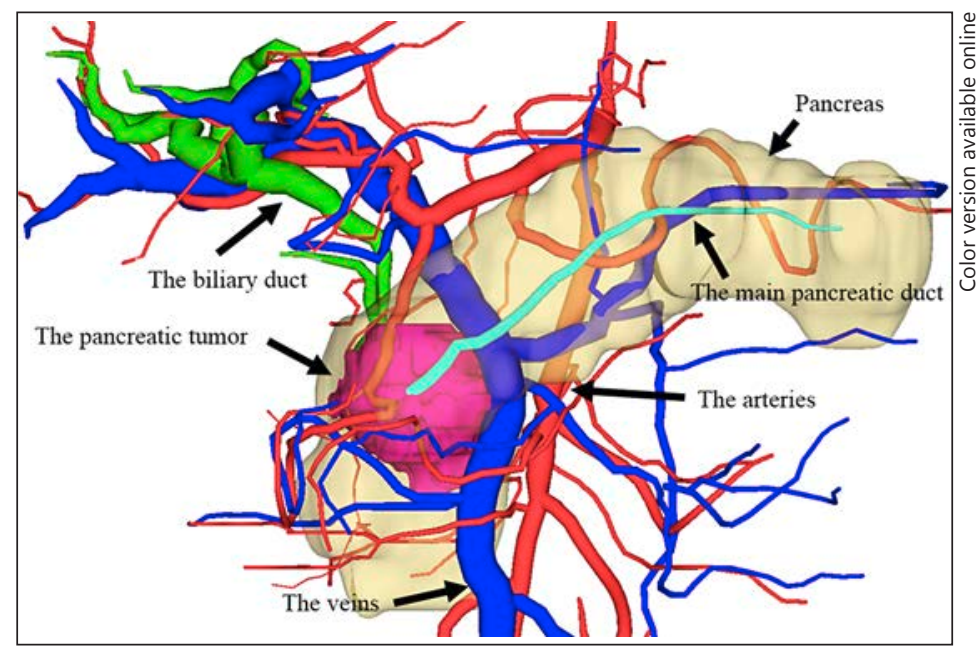

Surgical Procedures

The surgical procedures depend on the pancreatic location of the primary tumor. Among the 61 patients, 49 underwent subtotal stomach-preserving PD and modified Child's reconstruction $[11,12]$. The remaining 12 patients underwent DP. Systemic regional lymphadenectomy was performed in all cancer patients; the lymph nodes included in the dissection were those in the hepatoduodenal ligament, the posterior pancreaticoduodenal nodes, and the nodes along the common hepatic artery. In a case performed with 3D surgical simulation and navigation, the surgical team observed the preoperative simulated 3D image on a large display during the surgery. Therefore, the surgical team was able to communicate and discuss the critical points of the surgical procedure. All surgical procedures were performed under the supervision of one or two senior pancreatic surgeons.

\section{Statistical Analyses}

The correlation of the outcomes for surgeries performed prior to and after the adoption of 3D surgical simulations was analyzed using the $\chi^{2}$ test or Fisher's exact test, as appropriate.

Statistical analyses were performed using IBM SPSS (version 21; IBM, Armonk, NY, USA), and $p$ values $<0.05$ were considered statistically significant.

\section{Results}

\section{Patient Characteristics}

The characteristics of 61 patients who were subject to preoperative 3D surgical simulations and navigation are presented in Table 1 . The mean age was 68 years. There were 34 men and 27 women. In total, 24 patients were diagnosed with pancreatic cancer, 13 patients with intraductal papillary mucinous neoplasm, 11 patients with biliary cancer, 4 patients with pancreatic neuroendocrine tumor, 2 patients with duodenal cancer, and 7 patients with another diagnosis. Forty-nine patients received PD, and 12 patients received DP. Portal vein resection was performed in 5 patients. The mean value of the main duct diameter was 3.19 $\mathrm{mm}$.

\section{Questionnaires for 3D Simulation Images}

The overall mean score of all 181 self-assessment questionnaire responses was 5.33 . The mean scores were $4.29 \pm 2.11(n=85)$ and $7.45 \pm 1.45(n=96)$ for surgical residents in groups A and B, respectively. A statistically significant difference was observed between the two groups ( $p<0.001)$ (Fig. 2). 
Fig. 2. The self-assessment scores between the self-constructed 3D images and the simulated 3D images among surgical residents. A statistically significant difference was observed between the two groups.

Table 1. Characteristics of the patients who underwent 3D surgical simulation and navigation $(n=61)$
Miyamoto et al.: Impact of Three-Dimensional Surgical Simulation on Pancreatic Surgery

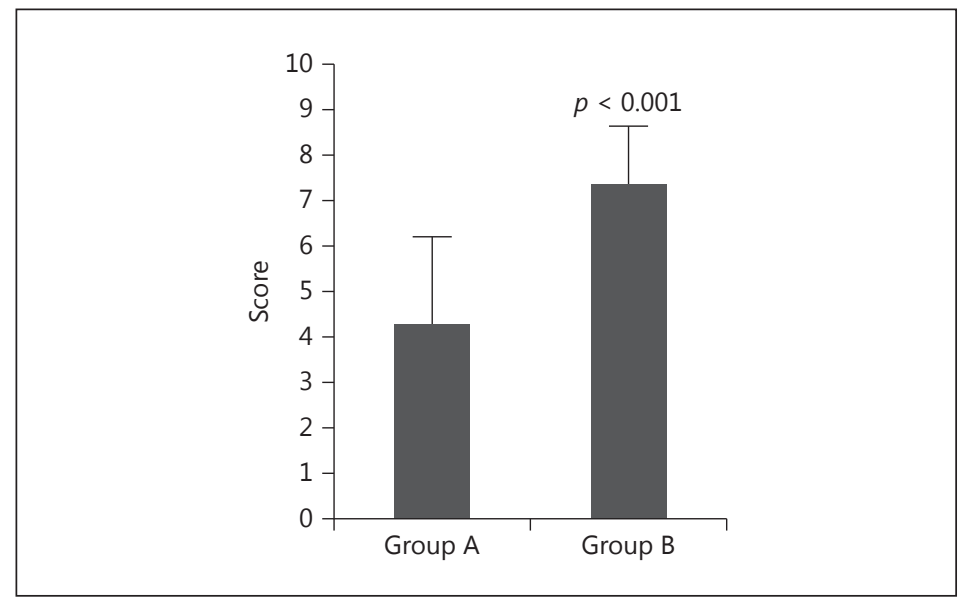

$\begin{array}{lc}\text { Age, years } & 68 \pm 12.4 \\ \text { Males/females } & 34 / 27 \\ \text { Primary disease } & \\ \quad \text { Pancreatic cancer } & 24 \\ \quad \text { IPMN } & 13 \\ \quad \text { Biliary cancer } & 11 \\ \quad \text { Pancreatic neuroendocrine tumor } & 4 \\ \quad \text { Duodenal cancer } & 2 \\ \quad \text { Others } & 7 \\ \text { Surgical procedure } & \\ \quad \text { Pancreaticoduodenectomy } & 49 \\ \quad \text { Distal pancreatectomy } & 12 \\ \text { Portal vein resection } & 5 \\ \text { Main pancreatic duct diameter } & 3.19 \pm 2.01\end{array}$

Data are presented as $n$ or mean \pm SD. 3D, three-dimensional; IPMN intraductal papillary mucinous neoplasm.

\section{Discussion}

In this study, the questionnaire survey revealed that the surgical residents' preoperative understanding of the anatomical MDCT images differed from the simulated 3D images by 30-60\%, and that the 3D surgical simulations were helpful for the surgical residents to understand anatomical images. Particularly, the most error-prone anatomical structure was the route of the arterial system, especially the hepatic artery. Therefore, we discussed the consistency between the surgical residents' 3D drawings and the 3D model in preoperative conference. We assumed that this discussion improved the self-constructed anatomical images created by the surgical residents. Based on the assessments stratified by the number of postgraduate years of experience, a tendency in which consistent scores increased as the postgraduate years advanced was noted. This result indicated that surgical experience is necessary for young residents to visualize the 3D anatomical structures from MDCT images. The improved understanding of the 3D anatomical images and the sharing of these images with the surgical staff was thought to contribute to reductions in operation time and intraoperative blood loss as well as the mastery of surgical techniques and advances in surgical instruments [5-7]. Furthermore, we assumed that the present 3D model was especially useful 
for cancer surgery, which required systemic regional lymphadenectomy. Improving the preoperative understanding of the 3D anatomic images, including the blood vessels and organs, as well as sharing the images with the surgical staff might contribute to safer and more radical cancer surgery.

The assessment method used in the present study (i.e., self-assessment by using a questionnaire) might be considered subjective. However, this method (i.e., repeated self-assessment by 15 young unskilled surgeons with regard to the 3D anatomical structure) was more appropriate for the evaluation of the contributions of 3D surgical simulation to the anatomical understanding than the assessment performed by skilled experts. In addition, this assessment method was also useful in the training of young surgeons, considering that surgical training is one of our missions other than ensuring safe surgery. In particular, pancreatic surgery requires surgeons to have a precise understanding of the surgical anatomy. It is difficult for young surgeons to have a precise anatomical knowledge of the region because they do not have sufficient surgical experience. We assumed that their 3D drawings from the MDCT and the selfassessment questionnaire after preoperative conference with reconstructed 3D images contributed to teaching the actual 3D anatomical relationships during pancreatic surgery.

Recent developments in MDCT technology, which provide rapidly acquired multiphase datasets in the early/delayed phase, allow for the continuous acquisition of the CT view. In 2001, Kamel et al. [13] reported the reconstruction of 3D images for surgical planning in potential donors, which were evaluated for adult living right lobe liver transplantation. Many institutes in Japan began to construct 3D images from MDCT datasets of patients undergoing hepatic resection to facilitate surgical planning and allow for the sharing of the complicated anatomical images with the surgical staff $[8,9,14,15]$. However, in biliary and pancreatic surgeries, information regarding the bile and pancreatic ducts is essential, but the 3D simulations from MDCT images appeared to be insufficient. We originally developed 3D images by integrating MDCT and MRCP images to produce accurate preoperative anatomical images, and we applied this method for the depiction of hepatic anatomy prior to hepato-biliarypancreatic surgery [10]. By integrating these two images, it is possible to better understand the anatomical relationships among the bile or pancreatic duct arrangements, vascular components, i.e. hepatic artery and portal vein, and the parenchymal organs, i.e. pancreas and liver. Moreover, preoperative sharing of the anatomical 3D images with the surgical staff became possible.

In conclusion, 3D surgical simulation was useful for understanding and sharing surgical information about pancreatic surgery, especially in younger postgraduate surgeons. We propose the use of 3D images as a new modality for preoperative assessment before pancreatic surgery. The present study has limitations. Because this was a single-center study with a relatively small patient cohort, these results will need to be prospectively confirmed by additional multicenter large-scale studies.

\section{Disclosure Statement}

The authors declare no conflicts of interest. 
Miyamoto et al.: Impact of Three-Dimensional Surgical Simulation on Pancreatic Surgery

\section{Appendix}

Self-Assessment Questionnaire

Please indicate your postgraduate year

In terms of the three points below, please indicate the anatomical differences between the 3D images and your self-drawn 3D image sketches on a 10-point scale, with 10 indicating 100\% anatomical consistency.

1. The route of the arterial system, especially the hepatic artery, the splenic artery, and the divergent patterns of the inferior pancreaticoduodenal artery (IPDA)

$(0 \cdot 1 \cdot 2 \cdot 3 \cdot 4 \cdot 5 \cdot 6 \cdot 7 \cdot 8 \cdot 9 \cdot 10)$

2. The route of the venous system, especially the left gastric vein, the inferior mesenteric vein, and the divergent patterns of the gastro-colic trunk

$(0 \cdot 1 \cdot 2 \cdot 3 \cdot 4 \cdot 5 \cdot 6 \cdot 7 \cdot 8 \cdot 9 \cdot 10)$

3. The tumor location and dissection line of the pancreas

$(0 \cdot 1 \cdot 2 \cdot 3 \cdot 4 \cdot 5 \cdot 6 \cdot 7 \cdot 8 \cdot 9 \cdot 10)$

\section{References}

1 DeOliveira ML, Winter JM, Schafer M, Cunningham SC, Cameron JL, Yeo CJ, Clavien PA: Assessment of complications after pancreatic surgery: a novel grading system applied to 633 patients undergoing pancreaticoduodenectomy. Ann Surg 2006;244:931-937.

2 Ward J, Sheridan MB, Guthrie JA, Davies MH, Millson CE, Lodge JP, Pollard SG, Prasad KR, Toogood GJ, Robinson PJ: Bile duct strictures after hepatobiliary surgery: assessment with MR cholangiography. Radiology 2004; 231:101-108.

3 Miyamoto R, Oshiro Y, Hashimoto S, Kohno K, Fukunaga K, Oda T, Ohkohchi N: Three-dimensional imaging identified the accessory bile duct in a patient with cholangiocarcinoma. World J Gastroenterol 2014;32: 11451-11455.

4 Miyamoto R, Oshiro Y, Nakayama K, Kohno K, Hashimoto S, Fukunaga K, Oda T, Ohkohchi N: Three-dimensional simulation of pancreatic surgery showing the size and location of the main pancreatic duct. Surg Today 2017;47:357-364.

5 Uchida M: Recent advances in 3D computed tomography techniques for simulation and navigation in hepatobiliary pancreatic surgery. J Hepatobiliary Pancreat Sci 2014;21:239-245.

6 Murakami K, Nakahara T: Efficacy of 3D-positron emission tomography/computed tomography for upper abdomen. J Hepatobiliary Pancreat Sci 2014;21:246-250.

7 Abe Y, Itano O, Kitago M, Shinoda M, Yagi H, Hibi T, Takano K, Chiba N, Kawachi S, Shimazu M, Kitagawa Y: Computer assisted surgery, preoperative planning and navigation for pancreatic cancer. J Hepatobiliary Pancreat Sci 2014;21:251-255.

8 Takahashi K, Sasaki R, Kondo T, Oda T, Murata S, Ohkohchi N: Preoperative 3D volumetric analysis for liver congestion applied in a patient with hilar cholangiocarcinoma. Langenbecks Arch Surg 2010;395:761-765.

9 Sasaki R, Kondo T, Oda T, Murata S, Wakabayashi G, Ohkohchi N: Impact of three-dimensional analysis of multidetector row computed tomography cholangioportography in operative planning for hilar cholangiocarcinoma. Am J Surg 2011;202:441-448.

10 Oshiro Y, Sasaki R, Nasu K, Ohkohchi N: A novel preoperative fusion analysis using three-dimensional MDCT combined with three-dimensional MRI for patients with hilar cholangiocarcinoma. Clin Imaging 2013;37:772-774.

11 Matsumoto I, Shinzeki M, Asari S, Goto T, Shirakawa S, Ajiki T, Fukumoto T, Suzuki Y, Ku Y: A prospective randomized comparison between pylorus- and subtotal stomach-preserving pancreatoduodenectomy on postoperative delayed gastric emptying occurrence and long-term nutritional status. J Surg Oncol 2014;109: 690-696.

12 Hayashibe A, Kameyama M, Shinbo M, Makimoto S: The surgical procedure and clinical results of subtotal stomach preserving pancreaticoduodenectomy (SSPPD) in comparison with pylorus preserving pancreaticoduodenectomy (PPPD). J Surg Oncol 2007;95:106-109.

13 Kamel IR, Kruskal JB, Pomfret EA, Keogan MT, Warmbrand G, Raptopoulos V: Impact of multidetector CT on donor selection and surgical planning before living adult right lobe liver transplantation. Am J Roentgenol 2001;176:193-200.

14 Mise Y, Tani K, Aoki T, Sakamoto Y, Hasegawa K, Sugawara Y, Kokudo N: Virtual liver resection: computer-assisted operation planning using a three-dimensional liver representation. J Hepatobiliary Pancreat Sci 2013;20:157-164.

15 Fang CH, Liu J, Fan YF, Yang J, Xiang N, Zeng N: Outcomes of hepatectomy for hepatolithiasis based on 3-dimensional reconstruction technique. J Am Coll Surg 2013;217:280-288. 